\title{
Avaliação de Personalidade Tipo D e Percepção de Doença em Pacientes Cardiopatas
}

\author{
Evaluation of Type D Personality and IIness Perception in Patientes With Coronary \\ Artery Disease
}

\author{
Evaluación de Personalidad Tipo D y Percepción de Enfermedad en Pacientes \\ Cardiopatas
}

\author{
Natan Pinto do Nascimento ${ }^{1}$ \\ Gabriela Woll Dias \\ Luciane Daroit \\ Ciomara Ribeiro Silva Benincá \\ Universidade de Passo Fundo
}

\begin{abstract}
Resumo
As doenças cardiovasculares representam a principal causa de morte no Mundo, podendo sua etiologia estar ligada a fatores psicológicos, como a Personalidade Tipo D. Indivíduos com esse tipo de personalidade apresentam vivência crônica de afetividade negativa associada à inibição social. Outro fator importante no adoecimento é a maneira como os sujeitos percebem sua saúde, pois esta resultará na implementação de estratégias para lidar com sua condição. Nesse sentido, o objetivo do trabalho foi avaliar, pela aplicação dos instrumentos Brief IPQ e DS-14, a relação entre a percepção de doença e a prevalência de Personalidade Tipo D em 80 pacientes com DAC, durante internação hospitalar. Na comparação das médias, pode-se verificar que os sujeitos com PTD tendem a perceber maior severidade em sua doença. Foram encontrados, também, índices baixos de entendimento dos fatores causais e temporalidade da doença. Ressalta-se o papel das estratégias psicoeducativas como fundamentais na atenção a esses pacientes.
\end{abstract}

Palavras-chave: personalidade, percepção de doença, distúrbios cardiovasculares, Psicologia

\begin{abstract}
Cardiovascular diseases are the main cause of death in Brazil, multiple factors are related to the etiology of this condition, including those of psychological origin, such as Type D Personality, that seems to predispose subjects to a chronic stress state that would increase the risk of developing CVDs. Another factor with influence in the disease is the way individuals perceive their health status, being this perception responsible for the way they create devices to deal with their condition. In this sense, the objective of the present study was to evaluate, using Brief IPQ and DS-14 Scale, the relationship between the perception of disease and the prevalence of Type D Personality in 80 patients with CAD during hospitalization. It can be observed that, although no statistical correlations were found between the variables, subjects with TDP tend to perceive greater severity in their disease. We also found low levels of understanding and the temporality of the disease. The role of psychoeducational strategies is highlighted.
\end{abstract}

Keywords: Type D personality, illness perceptions, cardiovascular disease, Psychology

\section{Resumen}

Las enfermedades cardiovasculares representan la principal causa de muerte en el mundo, pudiendo su etiología estar ligada a factores psicológicos, como la Personalidad Tipo D. Otro factor importante en los contextos de enfermedad es la manera en que los sujetos perciben su salud, ya que resultará en la implementación de estrategias para lidiar con su condición. En este sentido el objetivo del presente trabajo fue evaluar por la aplicación de los instrumentos Brief IPQ y DS-14, la relación entre la percepción de enfermedad y la prevalencia de Personalidad Tipo D en 80 pacientes con DAC durante internación hospitalaria. Aunque, no significativamente entre las variables, los sujetos con PTD tienden a percibir mayor severidad en su enfermedad. Se encontraron, también, índices bajos de entendimiento de los factores causales y de la temporalidad de la enfermedad. Se resalta el papel de las estrategias psicoeducativas como fundamentales en la atención a estos pacientes.

Palabras clave: personalidad Tipo D, percepción de enfermedad, cardiovascular, Psicología

${ }^{1}$ Endereço de contato: Rua José Vezaro, 100, Marau, RS, CEP 99150-000. E-mail: natannascimento@live.com 


\section{Introdução}

As doenças cardiovasculares (DCV) figuram entre as principais causas de morbimortalidade no mundo. Embora venha ocorrendo gradativa diminuição nas taxas de incidência (Feng et al., 2017) segundo Organização Mundial da Saúde (OMS), no ano de 2015, cerca de 17,5 milhões de pessoas morreram por DCVs. No Brasil, dados de 2013 do DATASUS revelam que mais de um milhão de pessoas internaram em hospitais em função de DCVs, e cerca de 100.000 pessoas morreram em decorrência da doença isquêmica do coração.

A etiologia multifatorial das DCVs é amplamente conhecida, com fatores de risco classificados em duas modalidades: A primeira diz respeito aos aspectos de predisposição não modificáveis, tais como hereditariedade, sexo (maior predisposição em homens dependendo da faixa etária), idade (superior a 45 anos); A segunda abarca os fatores de risco passíveis de intervenção e modificação, a saber, hipertensão arterial sistêmica (HAS), colesterol aumentado, tabagismo, sedentarismo, obesidade, diabetes mellitus (DM) e fatores psicossociais, como o estresse (Wittkopf, Souza, Ferrari, Medeiros, \& Cardoso, 2016; Gus et al., 2015).

A psicologia, levando em conta que determinados padrões de comportamentos podem estar associados a características de personalidade dos sujeitos, vem realizando, há mais de 20 anos, pesquisas com objetivo de desenvolver uma classificação da população em perfis psicológicos que possam estar relacionados à predisposição para desenvolvimento de doenças cardíacas (Denollet \& Brutsaert, 1998). Essa classificação teve como intuito auxiliar no desenvolvimento de estratégias de intervenção para a promoção de mudanças comportamentais desses sujeitos (Carver \& Connor-Smith, 2010).

Considerando o pressuposto acima, Denollet et al. (1996), através da análise de estudos prévios que definiam aspectos distintos de personalidade em pacientes com doença coronariana, verificaram estatisticamente alta correlação entre os traços de inibição social (IS) e afetividade negativa (AN). Foi verificado que a interação entre a vivência crônica de emoções negativas e a inibição da expressão desses sentimentos caracterizaria uma forma específica de estresse que poderia atuar como fator de predisposição dos indivíduos à manifestação ou exacerbação de problemas de saúde (Suguihura, 2014). No mesmo estudo, foi cunhado o termo "Type D", referente à palavra na língua inglesa "distress", que pode ser encontrada no dicionário como aflição ou angústia (Parker \& Stahel, 2010).

Tendo como base os dados verificados, foi proposto por Denollet (2005) um instrumento para mensuração desses traços de personalidade na população, a Type D Scale-14 (DS-14). Estudos baseados nessa avaliação, têm demonstrado uma prevalência variável de 20 a 50\% do Tipo de Personalidade entre pacientes com DCV (Annagür, Demir, Avci, \& Uygur, 2017; Al-Qezweny et al., 2016; Cao, Wang, Wong, Chow \& Chair, 2016; Doyle, McGee, Delaney, Motterlini, \& Conroy, 2011).

Batselé et al. (2017) define a personalidade Tipo D como um construto que se refere à combinação de dois traços: a AN e a IS, sendo compreendidos na subscala AN três traços predominantes de personalidade: disforia, ansiedade e irritabilidade; de igual forma, na subscala de IS: desconforto social, retração e falta de equilíbrio social. Kupper e Denollet (2018) afirmam que a AN é caracterizada pela tendência a vivência de emoções negativas como raiva, tristeza, medo e irritabilidade, ao longo do tempo e das situações, junto com a IS, que se refere à tendência ao não compartilhamento dessas emoções nas interações so- 
ciais em função do medo de rejeição ou desaprovação.

Alguns autores também sugerem que a Personalidade Tipo D (PTD) pode prever o início e a persistência de sintomas depressivos em pacientes com DAC, podendo ser relacionada, também, com a presença de sintomas depressivos na população geral e em pacientes com DM (Nefs et al., 2015; Doyle, McGee, Delaney, Motterlini, \& Conroy, 2011; Michal, Wiltink, Grande, Beutel, \& Brähler, 2011), além de servir como preditor de mortalidade, se comparada com a de pacientes não-PTD com doença cardíaca já estabelecida (Denollet et al. 1996). Lambertus et al. (2018) reforçam que, embora a Personalidade Tipo D seja considerada um fator de risco para doenças mentais e físicas, ela não equivale a um transtorno mental específico como a depressão, por exemplo. Ao contrário, o construto enfatiza o papel dos traços normais, e não os psicopatológicos.

Outro fator de influência no prognóstico das DCVs e que também serve para entendimento dos impactos causados na vida dos indivíduos, são as crenças que este possui em relação à própria doença (Altenhofen, Lima, \& Castro, 2016). Entende-se que os pacientes, quando confrontados com o diagnóstico de doenças, mostram padrões de crenças sobre a sua condição as quais, geralmente, atuarão como determinantes dos comportamentos utilizados para lidar com o adoecimento (Petrie \& Weinman, 2006).

Segundo o modelo de Leventhal, Nerenz e Steele (1984), chamado de Modelo de Auto Regulação em Saúde ou Modelo do Senso Comum (MSC), a percepção de doença pelo indivíduo envolve a representação cognitiva e emocional que este atribui ao seu adoecimento, sendo seguido pela busca e implementação de estratégias de enfrentamento para lidar com sua própria condição. As estratégias de enfrentamento, por sua vez, são fortemente influenciadas pelas percepções cognitivas e emocionais do adoecer, isto é, as vivências pessoais de cada sujeito interferem na percepção de sua própria doença e nas estratégias que serão utilizadas para lidar com sua condição de saúde. Uma revisão sistemática (Seabra, Peuker, \& Castro, 2015) que compilou as contribuições do MSC, constatou que as crenças desajustadas da doença podem impactar em alterações psicológicas, gerando ansiedade e depressão, e dificultar os comportamentos em saúde (autocuidado) e adesão ao tratamento.

Sobre essa questão, estudo realizado por Riley et al. (2012) utilizando o Ilness Perception Questionnaire (Brief-IPQ) verificou, nos pacientes que apresentavam crenças de consequências negativas advindas de sua doença, associação com piora no funcionamento físico e mental, níveis menores de qualidade de vida e maior predisposição à depressão. Maclnnes (2013) relacionou as crenças dos pacientes com maior entendimento de sua doença à meIhor adesão aos tratamentos propostos. Yu, Chen, Zhang e Liu (2011) demostraram também o papel desempenhado pela Personalidade Tipo D como influência negativa na percepção da doença, gerando maior consciência da severidade de sua condição em pacientes com DAC.

Nesse sentido, o objetivo do presente trabalho foi avaliar os valores atribuídos à percepção de doença e a prevalência de PTD em uma amostra de pacientes internados por complicações arteriais coronarianas, além de verificar, através de análises estatísticas, se existe relação entre as variáveis estudadas. 


\section{Metodologia}

Esta é uma pesquisa quantitativa, de caráter transversal e descritivo, com amostragem por conveniência. O projeto foi submetido ao Comitê de Ética e Pesquisa com Seres Humanos da Universidade de Passo Fundo (CEP), e a pesquisa foi iniciada após recebimento do parecer favorável sob o número 2092312.

\section{Participantes}

Participaram deste estudo 80 pacientes adultos, provenientes de municípios variados e de contextos socioculturais diversos, internados pela cardiologia em um hospital geral de uma cidade de porte médio no interior do estado do Rio Grande do Sul, no período de julho a setembro de 2017. Os critérios de inclusão foram os seguintes: a) estar hospitalizado pela cardiologia e possuir diagnóstico de DAC em curso; b) ter no mínimo 18 e no máximo 80 anos de idade; c) possuir condições cognitivas para entendimento dos procedimentos e objetivos da pesquisa; d) aceitar participar e assinar o Termo de Consentimento Livre e Esclarecido (TCLE).

Os dados sociodemográfios dos participantes encontram-se discriminados na Tabela 1 expressando valores em relação à frequência e porcentagem ou média e desvio padrão.

Tabela 1

Caracterização sociodemográfica

Características

\begin{tabular}{lrr}
\hline Sexo (n; \%) & 56 & 70 \\
$\quad$ Masculino & 24 & 30 \\
Feminino & & \\
\hline Estado Civil (n; \%) & 5 & 6,3 \\
$\quad$ Solteiro (a) & 53 & 66,3 \\
Casado (a) & 15 & 18,8 \\
$\quad$ Divorciado/Separado (a) & 5 & 6,3 \\
$\quad$ Viúvo (a) & & \\
\hline Ocupação (n; \%) & 8 & 10 \\
$\quad$ Desempregado & 42 & 52 \\
Trabalhando & 26 & 32,5 \\
Aposentado & 4 & 5 \\
$\quad$ Em Auxílio Doença & & \\
\hline Escolaridade (n; \%) & 43 & 53,8 \\
Fundamental Incompleto & 10 & 12,5 \\
Médio Incompleto & 17 & 21,1 \\
Médio Completo & 7 & 8,8 \\
$\quad$ Superior & & \\
\hline Renda (n; \%) & 9 & 11,3 \\
1 Salário Mínimo & 31 & 38,8 \\
2 Salários Mínimos & 26 & 32,5 \\
3 Salários Mínimos &
\end{tabular}

Verificou-se que majoritariamente a amostra era composta por pessoas do sexo masculino (70\%), com idade média de 62,50 ( \pm 9.09$)$, casados $(66,3 \%)$, ativos do ponto de vista la- 
boral (52\%), com escolaridade fundamental incompleta $(53,8 \%)$ e renda média de 2 salários mínimos (38,8\%).

Em relação à apresentação clínica da amostra, 87,75\% ( $n=67)$ apresentavam diagnóstico de Síndrome Coronariana Aguda, e 16,25\% (n=13), de Angina Estável.

Quanto aos fatores de risco, $80 \%(n=64)$ dos pacientes possuem histórico familiar para DCVs, 13,75\% ( $n=11)$ são tabagistas, 40\% ( $n=32$ ) são ex-tabagistas, e 46,25\% ( $n=37)$ nunca fumaram. A HAS foi observada em $85 \%(n=68)$ dos pacientes; o DM, em 37,5\% ( $n=30)$; a dislipidemia, em 18,8\% ( $n=15)$; a doença pulmonar obstrutiva crônica, em $8 \%(n=10)$; e a doença renal crônica, em $4 \%(n=5)$.

Quanto ao histórico clínico pregresso, 68,8\% ( $n=55)$ dos pacientes possuíam implantação de stent com a quantidade variada $1(34,5 \%)$ e $7(3,6 \%) ; 27,5 \%(n=22)$ utilizam algum tipo de medicação psiquiátrica; e 16,3\% ( $n=13)$ já haviam realizado acompanhamento psicológico em algum momento de sua vida.

\section{Instrumentos}

Para coleta de dados, foi utilizado o prontuário do paciente, junto com um questionário sociodemográfico elaborado exclusivamente para o estudo. A análise do prontuário teve por finalidade obter dados referentes ao diagnóstico e motivo de internação dos pacientes. O questionário sociodemográfico foi utilizado para caracterização dos participantes quanto às seguintes informações: sexo, idade, situação conjugal e familiar, escolaridade, situação laboral, renda, histórico familiar de doença cardíaca, uso de medicação psiquiátrica, histórico de atendimento psicológico e histórico de adições (tabagismo).

As variáveis estudadas foram medidas por dois instrumentos: a Type D Personality Scale (DS14) e o Brief IIIness Perception Questionnaire (Brief IPQ).

A Type D Personality Scale (DS14), originalmente desenvolvida por Dennolet (2005), foi traduzida e validada para a população brasileira por Suguihura (2014) e avalia a vivência de Afetividade Negativa (AN) e Inibição Social (IS), através de 14 afirmativas para as quais o indivíduo possui cinco opções de resposta (0-5), em uma escala do tipo Likert. Pontuações iguais ou superiores a 10 pontos em ambas as subescalas são classificados como PTD. As subescalas da DSD-14 apresentaram bom em estabilidade temporal, com índice de correlação ( $r$ de Pearson) forte para AN $(0,76)$ e moderado para IS $(0,65)$. Com relação à consistência interna ( $\boldsymbol{\alpha}$ de Cronbach), ambas podem ter seus valores considerados altos: 0,79 para AN e 0,76 para IS.

O Brief IIIness Perception Questionnaire (BriefIPQ): originalmente proposto por Broadbent Petrie, Main, e Weinman, (2006) e traduzido no país por Nogueira (2012), é utilizado de forma a fornecer uma medida breve da percepção de doença. É composto por nove itens, dos quais três avaliam a representação cognitiva da doença; quatro, a representação emocional, através de uma escala do tipo Likert de 11 pontos (0-10) O alfa de Cronbach da versão brasileira referente aos sete itens é igual a 0,64; e duas perguntas abertas que avaliam a percepção acerca da dimensão temporal da enfermidade e a representação causal (três opções) do desenvolvimento da doença. $\mathrm{O}$ escore global do instrumento é expresso de 0 a 70: escores elevados indicam maior percepção de ameaça imposta pela enfermidade. 


\section{Procedimentos}

De posse do documento de autorização da equipe administrativa do hospital e da aprovação do CEP, os pacientes foram identificados conforme critérios de inclusão e convidados a participar da pesquisa mediante leitura e assinatura do TCLE. A coleta dos dados foi realizada em forma de entrevista à beira do leito do paciente, nas dependências do hospital, em dois momentos distintos: avaliação e devolução.

Avaliação: preenchimento do questionário sociodemográfico e aplicação dos instrumentos DS-14 e Brief IPQ. Nesse mesmo contato, o paciente era orientado pelos pesquisadores sobre a devolução dos resultados.

Devolução: realizado após a análise e correção dos instrumentos, consistia em duas formas de abordagem distintas. Na primeira, quando da verificação de escores confirmatórios de PTD, percepções negativas sobre a condição de saúde, concepções errôneas ou desconhecimento dos fatores de risco e causais das doenças, tinha como objetivo a realização de psicoeducação. Nesse momento, era realizado também orientações, encaminhamento e reforço da procura por psicoterapia nos casos específicos. A segunda forma de abordagem, tendo apenas caráter informativo, era realizada com pacientes que não apresentaram escores expressivos em nenhuma das variáveis.

Os resultados foram analisados pelo Statistical Package for the Social Sciences-SPSS 20, sendo as variáveis categóricas expressas como frequência absoluta e percentis, e as relativas como média \pm desvio padrão. A análise de relação entre as variáveis foi realizada através do Teste de Correlação de Pearson, adotando-se um nível de significância de $p<0,05$.

\section{Resultados}

Os resultados serão apresentados em formas descritiva levando em conta o levantamento da DS-14 (Tabela 2), do Brief IPQ (Tabela 3 e Tabela 4) e a correlação de variáveis.

\section{Tabela 2}

Frequência da Personalidade Tipo D no DS-14

Frequência (\%)
n (\%) Feminina
n (\%) Masculina

\begin{tabular}{cc}
\hline Personalidade Tipo D & Personalidade Tipo Não D \\
\hline $33(41,25)$ & $47(58,75)$ \\
$9(37,5)$ & $15(62,5)$ \\
$24(42,9)$ & $32(57,1)$
\end{tabular}

Conforme verificado na tabela, 41,25\% ( $n=33$ ) dos participantes apresentam pontuações $\geq 10$ em ambas as subescalas de AN e IS, fechando assim, critérios para PTD, sendo a maioria indivíduos do sexo masculino $(n=24)$. Quando comparada a de prevalência de PTD de acordo com o sexo, não se verificam diferenças significativas entre mulheres $(37,5 \%)$ e homens $(42,9 \%)$. 
Tabela 3

Dimensões de representação da doença cardíaca no Brief IPQ

\begin{tabular}{lcc}
\hline Dimensões & Média & DP \\
\hline Consequências & 7,2 & 2,4 \\
Dimensão Temporal & 4,68 & 3,82 \\
Controle Individual & 0,8 & 1,8 \\
Controle do Tratamento & 3,1 & 2,6 \\
Preocupação & 7,5 & 3,1 \\
Emoções & 3,4 & 2,6 \\
Compreensão da Doença & 3,2 & 3,1
\end{tabular}

No presente estudo, como se pode observar, as médias mais elevadas obtidas no questionário de percepção de doença foram relativas à representação emocional da doença na dimensão preocupação $(7,5 \pm 3,1)$ e na representação cognitiva em relação às consequências da doença $(7,2 \pm 2,4)$, a menor média foi encontrada em relação ao controle individual da doença $(0,8 \pm 1,8)$.

A dimensão de percepção temporal da doença é dada por uma pergunta aberta. As respostas dos participantes foram agrupadas em quatro categorias: 1$)$ a dos $37,5 \%(n=30)$ dos participantes que acreditam que seu problema de saúde estará resolvido após a realização de procedimento (angioplastia, cirurgia e tratamento medicamentoso); 2) a dos 36,25\% ( $n=29$ ) que relacionam seu problema de saúde à uma condição crônica; 3 ) a dos $13,75 \%(n=11)$ que responderam não saber ou não ser capaz de precisar estimativa de duração de sua condição de saúde; 4) a dos 3,75\% ( $n=3)$ que apresentaram respostas de caráter religioso, delegando a uma entidade superior o conhecimento da duração de sua doença.

Os dados em relação à causalidade da doença cardíaca foram classificados em categorias e estão discriminados abaixo na Tabela 3:

Tabela 4

Fatores relacionados à causalidade da doença cardíaca no Brief IPQ

\begin{tabular}{lrlrlr}
\hline \multicolumn{1}{c}{ 10 fator causal } & \multicolumn{1}{c}{ \% } & 2o fator causal & \multicolumn{1}{c}{ \% } & 3o fator causal & \multicolumn{1}{c}{ \% } \\
\hline Emocional & 18,75 & Não sabe & 30 & Não sabe & 61,25 \\
Alimentação & 16,25 & Emocional & 22,5 & Alimentação & 12,5 \\
Tabagismo & 15 & Alimentação & 10 & Hábitos de Vida & 10 \\
Hereditariedade & 13,75 & Tabagismo & 10 & Emocional & 5 \\
Não sabe & 10 & Hábitos de vida & 8,75 & Hereditariedade & 5 \\
Outros & 8,75 & Alcoolismo & 6,25 & Trabalho & 3,75 \\
Trabalho & 7,5 & Hereditariedade & 6,25 & Alcoolismo & 1,25 \\
Hábitos de Vida & 6,25 & Outros & 3,75 & Tabagismo & 1,25
\end{tabular}

Conforme os dados acima, verificou-se alta prevalência dos fatores atribuídos às questões emocionais como causadoras da doença, assim como altas taxas de desconhecimento dos fatores causais, observando-se também taxas moderadas atribuídas à má alimentação, tabagismo, hereditariedade e hábitos de vida não saudáveis. 
Sobre a correlação entre as variáveis, não foi verificada significante associação estatística no teste de correlação de Pearson entre as variáveis de percepção de doença, inibição social e afetividade negativa, tanto para indivíduos com PTD (IPQXAF p=0,7085; IPQxIS p =0,9042), quanto para os não-PTD (IPQXAF p=0,8201; IPQxIS p=0,8751). Todavia os pacientes com PTD demonstraram médias mais elevadas em relação à percepção de doença $(30,82 \pm 6,79$ verso $28,53 \pm 5,33$ ) em comparação aos pacientes não-PTD.

\section{Discussão}

Os traços de personalidade vêm sendo estudados pela psicologia em relação ao impacto causado nos processos de adoecimento dos indivíduos (Thomas \& Castro, 2012). Data da década de 50 o constructo da personalidade Tipo A, o qual propunha que pacientes com um padrão de comportamento impaciente, competitivo e hostil possuiriam maiores chances de sofrerem um evento cardíaco (Friedman, 1977). No estudo realizado por Grossarth-Maticek, Eysenck e Vetter (1988), com a finalidade de classificação dos entrevistados em quatro perfis, sendo um destes o Tipo II- reação à frustração com raiva, agressividade e excitação emocional- foi encontrada maior probabilidade de morte dos indivíduos desse grupo por DCVs.

Denollet, Sys e Brutsaert (1995), baseados nesses estudos anteriores, apresentam o conceito de PTD relacionando os traços de personalidade de vivência de afetividade negativa e inibição social à geração de um estado de estresse que estaria ligado à manifestação ou exacerbação de doenças. O entendimento da forma como a PTD exerce influência nos sujeitos ainda é escasso, pesquisas têm relacionado seus efeitos em relação à desregulação dos níveis de cortisol, com aumento nos níveis de biomarcadores inflamatórios, e disfunção edotelial (Van Doreen et al, 2016; Granville Smith, Parker, Cvejic, \& Vollmer-Conna, 2015).

Procurando-se investigar a prevalência da Personalidade tipo D em uma amostra de pacientes internados por complicações cardiovasculares, o presente estudo demostrou uma prevalência de $41,25 \%$ de pacientes cardíacos com esse tipo de personalidade, dados esses que corroboram com as pesquisas mais recentes que trazem prevalências entre 23,5\% e 46,5\% em pacientes com síndrome metabólica, DM, aterosclerose, pós-revascularização do miocárdio e com DCV instalada (Demirci, Yıldız, Selvi, \& Akpınar, 2016; Milicevic, Jaksic, Aukst-Margetic, \& Jakovljevic, 2015; Ogińska-Bulik, 2014; Sumin et al., 2012; 2015; Sumin \& Raih, 2016; Tziallas et al., 2011).

O conceito de personalidade é amplo na literatura, embora pareça haver uma concordância no sentido de definir a personalidade como algo característico dos sujeitos, que os torna únicos e orienta seus padrões de comportamento (Mendes, 2016). Quanto a PTD, sua influência nos processos de doença explica-se pela interação dos traços de personalidade, IS e AF, que a compõe (Nobre, Reis, Torres, \& Alchieri, 2011).

A afetividade negativa pode ser definida como a tendência à vivência contínua de estados de disforia e sentimentos como ansiedade, apreensão e irritabilidade e visão negativa de si próprio (Suguihura, 2014; Urso-Júnior, 2011).

A inibição social atuaria como fator de predisposição a experimentar sentimentos de tensão, insegurança e inibição no contato com outras pessoas, resultando em menor busca por suporte social, interações sociais de baixa qualidade, baixa autoestima e deliberada baixa expressão de afetos (Suguihura, 2014; Urso-Júnior, 2011). 
A interação entre esses dois fatores caracterizaria um sujeito com maiores dificuldades emocionais e de relacionamento interpessoal, aumentando a vulnerabilidade a estados de depressão e ansiedade, a exposição a comportamentos e hábitos de risco como o uso de álcool e tabaco, além de menor propensão à procura por atenção médica para suas queixas de saúde (Guimarães, 2016; Suguihura, 2014).

Conforme apontado no estudo de Eysenck (1996), não se pode comprovar que esses fatores psicológicos, isoladamente, atuem como causadores das doenças. O que se propõe, então, é a interpretação de que os agravos sejam resultado da interação de múltiplos fatores, entre eles os relacionados com a personalidade. Em relação aos fatores que atuam no estabelecimento e agravo das DCVs, a relação multicausal é bem descrita, sendo o estudo de Framingham o primeiro a descrever a importância de fatores como HAS, DM e tabagismo com o risco de estabelecimento de DCVs.

No presente estudo, pode-se constatar, ainda, a elevada prevalência de variados fatores de risco para doença cardíaca nos participantes. Do total da amostra, 13,75\% são constituídos por tabagistas ativos, e $40 \%$ possuíam histórico de uso de tabaco. A relação do uso de tabaco com as DCVs é fortemente descrita na literatura mundial; Mainali, Pant, Rodriguez, Deshmukh, e Mehta (2015) referem que as evidências científicas ligando o uso do cigarro às DCVs começaram surgir em meados dos anos 60. Pesquisas atuais definem o uso do tabaco como o fator de risco evitável mais importante para desenvolvimento de DAC (Carter et al., 2015), além de atuar como potencializador dos efeitos da dislipidemia e da resistência à insulina (Gepner et al., 2011).

Outros fatores de risco de grande prevalência na amostra foram a HAS (85\%) e o DM (37,5\%). A prevalência de HAS identificada neste estudo foi maior do que as taxas encontradas em pesquisas nacionais anteriores (Freitas et al., 2014; Poffo et al., 2017; Salomão et al., 2017). Já as taxas de DM não apresentaram variância significativa nos estudos (Wajner et al., 2017; Sousa, Bernardino, Vicelli, \& Kalinowski, 2014). Os dados discrepantes podem ser explicados utilizando dados da pesquisa conduzida por Macinko, Leventhal e Lima-Costa (2018), a qual relata altas taxas de subdiagnóstico dessa condição no Brasil, segundo esse estudo, de aproximadamente 51 milhões de indivíduos com HAS, quase 20 milhões desconheciam seu status de saúde embora realizassem acompanhamento médico regular.

No que tange ao prognóstico das doenças, é importante considerar a percepção que o indivíduo tem de sua própria condição, pois, segundo Cameron (2003), o paciente responde às ameaças impostas pela doença de acordo com as suas crenças pessoais, que influenciam na avaliação e interpretação sobre a relação saúde/doença. Na presente pesquisa, pôde-se verificar, através do Brief IPQ, que 30\% ( $n=24)$ dos participantes apresentaram a percepção de doença mais focada na ameaça,

Com relação às dimensões avaliadas pelo instrumento, o item "Preocupação", ligado à representação emocional da doença, e o item "Consequências", relacionado com a representação cognitiva, apresentaram as maiores médias $(7,5 \pm 3,1$ e 7,2 $\pm 2,4)$. A dimensão preocupação está relacionada com as angústias e receios que a doença provoca no doente (Pereira, Pedras, \& Machado, 2012). A dimensão consequências, engloba as concepções que o indivíduo possui a respeito da gravidade da doença e os efeitos que ela terá sobre seu funcionamento biopsicossocial (Nogueira, 2012). 
As médias mais baixas foram encontrados nas seguintes dimensões do instrumento: "Controle do Tratamento" (3,1 $\pm 2,6)$ e "Controle Individual" (0,8 \pm 1,8). Nogueira (2012), relata que existe uma inter-relação lógica entre os fatores da representação cognitiva das doenças, em que uma percepção maior das consequências da doença, conforme verificado nesta amostra, se relaciona com crenças mais frágeis quanto à possibilidade de cura e controle da doença.

Nesta amostra, os fatores causais atribuídos pelos indivíduos ao seu adoecimento estiveram relacionados com fatores externos e independentes dos comportamentos individuais, tais como estresse e outros fatores emocionais. Pode-se perceber, também altos índices de desconhecimento dos fatores causais do adoecimento. Fatores estes que podem estar refletidos nos valores de média baixos verificados no quesito "Compreensão" $(3,2 \pm 3,1)$.

A dimensão temporal de percepção da doença dividiu-se entre $37,5 \%$ dos pacientes com percepção de sua doença como uma condição aguda, os quais acreditavam na cura de sua enfermidade após realização de procedimentos como a angioplastia ou a otimização medicamentosa hospitalar, e em $36,25 \%$ dos pacientes que possuíam noção da condição crônica de seu problema de saúde. Salienta-se que as crenças causais são importantes em relação às estratégias que os indivíduos lançarão mão para lidar com sua condição (Gama, Mussi, Pires, \& Guimarães, 2012).

Nesse sentido, quando da identificação, por parte dos pesquisadores, durante a coleta de dados, de crenças equivocadas quanto às causas e a temporalidade de sua condição, foi realizada uma intervenção psicoeducativa com a finalidade de possibilitar ao paciente maior entendimento de seu quadro de saúde. As estratégias de educação com foco em saúde têm se mostrado válidas quando da promoção da ampliação do conhecimento dos sujeitos, buscando maior adesão, participação e controle de seu tratamento (Lemes \& Neto, 2017; Ferretti, Gris, Mattiello, Paz Arruda Téo, \& De Sá, 2015).

Quanto à tentativa de estabelecimento de relações estatísticas entre o fator de PTD e a percepção de doença, não foram obtidos resultados que demostrem significância estatística. Entretanto a análise das médias de percepção de doença dos pacientes com PTD mostraram-se maiores do que as dos pacientes não PTD, apoiando, assim, os dados encontrados por Yu et al. (2011) em que não puderam ser estabelecidas relações significativas entre as variáveis. Esse mesmo estudo demostrou média superior quanto à autoavaliação de severidade da doença cardíaca nos pacientes com PTD.

\section{Conclusões}

Embora não tenha sido possível demostrar correlações entre as variáveis estudadas, pode-se verificar que pacientes com PTD tendem a perceber maior severidade em relação à sua doença do que pacientes não-PTD, isso pode ser atribuído à interação dos traços de personalidade de AF e IS que constituem a PTD.

Pode-se perceber níveis de percepção de doença focada na ameaça por uma grande parte dos avaliados, isto significa uma consciência elevada em relação aos danos físicos e emocionais advindos do adoecimento.

Outro achado importante diz respeito às crenças dos pacientes quanto à baixa compreensão de sua condição de saúde e desconhecimento dos fatores causais. A literatura pes- 
quisada denota que esses fatores possuem influência negativa nas estratégias que os indivíduos utilizarão para lidar com as suas condições de saúde. Além disso, pode-se perceber dificuldades na adesão aos tratamentos propostos e autorregulação dos sujeitos em relação à exposição a fatores de risco, quando estes não são percebidos como danosos ou com influência no adoecimento.

O conhecimento dessas características, que possam interferir no curso da doença, em relação aos pacientes cardíacos deve servir como base para proposição de estratégias de intervenção voltadas à educação em saúde, tais quais as empregadas neste trabalho. $O$ enfoque em ações de educação voltadas à saúde tem como objetivos criar uma consciência crítica e autonomia nos sujeitos. Para isto, a escuta ativa e o diálogo aberto são fundamentais, já que essa prática busca não apenas que os sujeitos compreendam seus problemas de saúde, mas requer-Ihes encontrar soluções para lidar com estes de forma efetiva e perdurável (Taddeo et al., 2012).

Nesse sentido, o empoderamento dos sujeitos em relação à sua condição de saúde tem como finalidade construir com o sujeito estratégias mais adaptativas a sua condição, além de melhorar o engajamento nos tratamentos propostos. Nesse sentido, as estratégias psicoeducativas precisam levar em conta não só as informações concretas em relação às doenças, mas também as crenças que as pessoas possuem a respeito delas, sendo estas estratégias muito mais eficazes quando elaboradas dentro do contexto cultural e social do público que se quer alcançar (Castro, Teixeira, \& Duarte, 2017).

Dentre as limitações deste estudo, podemos destacar certa homogeneidade na população estudada em relação a fatores como a escolaridade, assim como o número amostral, sendo sugeridas novas pesquisas com populações mais diversas e de maior representatividade estatística das doenças cardiovasculares. Em relação à percepção de doença, podemos pensar também sobre o quanto o fato de estar hospitalizado, ou seja, em vivência plena dos sintomas da doença, possa influenciar em sua percepção negativa; sugere-se também que, em novos estudos, sejam incluídas na análise pessoas que não estejam necessariamente hospitalizadas em função da sua doença, pretendendo assim, diminuir a influência desse viés.

\section{Referências}

AL-Qezweny, M. N. A., Utens, E. M. W. J., Dulfer, K., Hazemeijer, B. A. F., van Geuns, R.-J., Daemen, J., \& van Domburg, R. (2016). The association between type D personality, and depression and anxiety ten years after PCl. Netherlands Heart Journal, 24(9), 538-543. doi: 10.1007/s12471-016-0860-4

Altenhofen, V., Lima, N. B. de, \& Castro, E. K. de. (2016). Percepção da doença em pacientes cardíacos: uma revisão sistemática. Estudos Interdisciplinares em Psicologia, 7(2), 45-63. doi: 10.5433/2236-6407.2016v7n2p45

Annagür, B. B., Demir, K., Avci, A., \& Uygur, Ö. F. (2017). Impact of a Type D Personality on Clinical and Psychometric Properties in a Sample of Turkish Patients With a First Myocardial Infarction.Journal of PsychiatricPractice, 23(1),3-10.doi:10.1097/PRA.0000000000000201

Batselé, E., Denollet, J., Lussier, A., Loas, G., Vanden Eynde, S., Van de Borne, P., \& Fantini-Hauwel, C. (2017). Type D personality: application of DS14 French version in general and clinical populations. Journal of Health Psychology, 22(8), 1075-1083. doi: 
$10.1177 / 1359105315624499$

Broadbent, E., Petrie, K. J., Main, J., \& Weinman, J. (2006). The Brief Illness Perception Questionnaire. Journal of Psychosomatic Research, 60(6), 631-637. doi: 10.1016/j.jpsychores.2005.10.020

Cameron, L. D. (2003). Conceptualizing and Assessing Risk Perceptions: A Self-Regulatory Perspective. In National Cancer Institute workshop on conceptualizing and measuring risk perception (pp. 1-12). Disponível em http://citeseerx.ist.psu.edu/viewdoc/ download?doi=10.1.1.520.5228\&rep=rep1\&type=pdf

Castro, E. K., Teixeira, V., \& Duarte, M. Q. (2017). Elaboração e avaliação de material educativo sobre a prevenção do câncer de mama. Mudanças-Psicologia da Saúde, 25(2), 51-57. Disponível em https://www.metodista.br/revistas/revistas-ims/index.php/MUD/article/ view/7461/6005

Cao, X., Wang, X.-H., Wong, E. M., Chow, C. K., \& Chair, S. Y. (2016). Type D personality negatively associated with self-care in Chinese heart failure patients. Journal of Geriatric Cardiology : JGC, 13(5), 401-7. doi: 10.11909/j.issn.1671-5411.2016.05.011

Carter, B. D., Abnet, C. C., Feskanich, D., Freedman, N. D., Hartge, P., Lewis, C. E., . . Jacobs, E. J. (2015). Smoking and Mortality- Beyond Established Causes. New England Journal of Medicine, 372(7), 631-640. doi: 10.1056/NEJMsa1407211

Carver, C. S., \& Connor-Smith, J. (2010). Personality and Coping. Annual Review of Psychology, 61(1), 679-704. doi: 10.1146/annurev.psych.093008.100352

Demirci, K., Yıldız, M., Selvi, C., \& Akpınar, A. (2016). The relationship between childhood trauma and type D personality in university students. International Journal of Social Psychiatry, 62(6), 542-548. doi: 10.1177/0020764016653774

Denollet, J. (2005). DS14: Standard Assessment of Negative Affectivity, Social Inhibition, and Type D Personality. Psychosomatic Medicine, 67(1), 89-97. doi: 10.1097/01.psy.0000149256.81953.49

Denollet, J., \& Brutsaert, D. L. (1998). Personality, disease severity, and the risk of long-term cardiac events in patients with a decreased ejection fraction after myocardial infarction. Circulation, 97(2), 167-73. Disponível em http://www.ncbi.nlm.nih.gov/pubmed/9445169

Denollet, J., Sys, S. U., \& Brutsaert, D. L. (1995). Personality and mortality after myocardial infarction. Psychosomatic Medicine, 57(6), 582-591. Disponível em http://www.ncbi.nlm.nih.gov/pubmed/8600485

Denollet, J., Sys, S. U., Stroobant, N., Rombouts, H., Gillebert, T. C., \& Brutsaert, D. L. (1996). Personality as independent predictor of long-term mortality in patients with coronary heart disease. Lancet (London, England), 347(8999), 417-21. Disponível em http://www.ncbi.nlm.nih.gov/pubmed/8618481

Doyle, F., McGee, H., Delaney, M., Motterlini, N., \& Conroy, R. (2011). Depressive vulnerabilities predict depression status and trajectories of depression over 1 year in persons with acute coronary syndrome. General Hospital Psychiatry, 33(3), 224-231. doi: 10.1016/j.genhosppsych.2011.03.008

Eysenck, H. I. (1996). Personality and crime: Where do we stand. Psychology, Crime \& Law, 2(3), 143-152. doi: 10.1080/10683169608409773

Feng, J.-L., Nedkoff, L., Knuiman, M., Semsarian, C., Ingles, J., Briffa, T., \& Hickling, S. (2017). 
Temporal Trends in Sudden Cardiac Death From 1997 to 2010: A Data Linkage Study. Heart, Lung and Circulation, 26(8), 808-816. doi: 10.1016/j.hlc.2016.11.021

Ferretti, F., Gris, A., Mattiello, D., Paz Arruda Téo, C. R., \& De Sá, C. (2015). Impacto de programa de educação em saúde no conhecimento de idosos sobre doenças cardiovasculares. Revista de Salud Pública, 16(6), 807-820. doi: 10.15446/rsap.v16n6.40165

Freitas, E. D. O., Nogueira, R. D. S., Stekel, L. M. C., Bublitz, S., Kirchhof, R. S., \& Guido, L. D. A. (2014). Perfil de pacientes com doença arterial coronariana submetidos ao cateterismo cardíaco. Revista de Enfermagem Da UFSM, 3, 679-688. doi: 10.5902/2179769211124

Friedman, M. (1977). Type A behavior pattern: some of its pathophysiological components. Bulletin of the New York Academy of Medicine, 53(7), 593-604. Disponível em http://www.ncbi.nlm.nih.gov/pubmed/196708

Gama, G. G. G., Mussi, F. C., Pires, C. G. da S., \& Guimarães, A. C. (2012). Crenças e comportamentos de pessoas com doença arterial coronária. Ciência \& Saúde Coletiva, 17(12), 3371-3383. doi: 10.1590/S1413-81232012001200022

Gepner, A. D., Piper, M. E., Johnson, H. M., Fiore, M. C., Baker, T. B., \& Stein, J. H. (2011). Effects of smoking and smoking cessation on lipids and lipoproteins: Outcomes from a randomized clinical trial. American Heart Journal, 161(1), 145-151. doi: 10.1016/j.ahj.2010.09.023

Granville Smith, I., Parker, G., Cvejic, E., \& Vollmer-Conna, U. (2015). Acute coronary syndromeassociated depression: The salience of a sickness response analogy? Brain, Behavior, and Immunity, 49, 18-24. doi: 10.1016/j.bbi.2015.02.025

Grossarth-Maticek, R., Eysenck, H. J., \& Vetter, H. (1988). Personality type, smoking habit and their interaction as predictors of cancer and coronary heart disease. Personality and Individual Differences, 9(2), 479-495. doi: 10.1016/0191-8869(88)90125-0

Guimarães, T. B. (2016). Perfil psicossocial de portadores de CDI: COMFORT-CDI. Tese de Doutorado, Faculdade de Medicina, Universidade de São Paulo, São Paulo. Disponível em http://www.teses.usp.br/teses/disponiveis/5/5131/tde-24102016-154635/

Gus, I., Ribeiro, R. A., Kato, S., Bastos, J., Medina, C., Zazlavsky, C., . . . Gottschall, C. A. M. (2015). Variations in the Prevalence of Risk Factors for Coronary Artery Disease in Rio Grande do Sul-Brazil: A Comparative Analysis between 2002 and 2014. Arquivos Brasileiros de Cardiologia, 105(6). doi: 10.5935/abc.20150127

Kupper, N., \& Denollet, J. (2018). Type D Personality as a Risk Factor in Coronary Heart Disease: a Review of Current Evidence. Current cardiology reports, 20(11), 104. doi: 10.1007/s11886-018-1048-x

Lambertus, F., Herrmann-Lingen, C., Fritzsche, K., Hamacher, S., Hellmich, M., Jünger, J., . . . \& Vitinius, F. (2018). Prevalence of mental disorders among depressed coronary patients with and without Type D personality. Results of the multi-center SPIRR-CAD trial. General hospital psychiatry, 50, 69-75. doi: 10.1016/j.genhosppsych.2017.10.001

Lemes, C. B., \& Neto, J. O. (2017). Aplicações da psicoeducação no contexto da saúde. Temas em Psicologia, 25(1), 17-28. doi: 10.9788/TP2017.1-02

Macinko, J., Leventhal, D. G. P., \& Lima-Costa, M. F. (2018). Primary Care and the Hypertension

Care Continuum in Brazil. Journal of Ambulatory Care Management, 41(1), 34-46. doi: 10.1097/JAC.0000000000000222

MacInnes, J. (2013). Relationships between illness representations, treatment beliefs and 
the performance of self-care in heart failure: a cross-sectional survey. European Journal of Cardiovascular Nursing, 12(6), 536-543. doi: 10.1177/1474515112473872

Mainali, P., Pant, S., Rodriguez, A. P., Deshmukh, A., \& Mehta, J. L. (2015). Tobacco and Cardiovascular Health. Cardiovascular Toxicology, 15(2), 107-116. doi: 10.1007/s12012-014-9280-0

Mendes, M. D. R. (2016). Relação entre traços de personalidade e as dimensões básicas dos estilos de vinculação em adultos (Tese de mestrado). Disponível em http://repositorio.ul.pt/handle/10451/27557

Michal, M., Wiltink, J., Grande, G., Beutel, M. E., \& Brähler, E. (2011). Type D personality is independently associated with major psychosocial stressors and increased health care utilization in the general population. Journal of Affective Disorders, 134(1-3), 396-403. doi: 10.1016/j.jad.2011.05.033

Milicevic, R., Jaksic, N., Aukst-Margetic, B., \& Jakovljevic, M. (2015). Personality traits and treatment compliance in patients with type 2 diabetes mellitus. Psychiatria Danubina, 27, (Suppl 2), 586-9. Disponível em http://www.ncbi.nlm.nih.gov/pubmed/26657986

Nefs, G., Speight, J., Pouwer, F., Pop, V., Bot, M., \& Denollet, J. (2015). Type D personality, suboptimal health behaviors and emotional distress in adults with diabetes: Results from Diabetes MILES-The Netherlands. Diabetes Research and Clinical Practice, 108(1), 94105. doi: 10.1016/j.diabres.2015.01.015

Nobre, T. T. X., Reis, L. A. dos, Torres, G. de V., \& Alchieri, J. C. (2011). Aspectos da personalidade e sua influência na percepção da dor aguda em pacientes submetidos à cirurgia cardíaca. Jornal Brasileiro de Psiquiatria, 60(2), 86-90. doi: 10.1590/S0047-20852011000200003

Nogueira, G. (2012, February 8). Adaptação e validação do Brief Illness Perception Questionnaire (Brief IPQ) para a cultura brasileira (Dissertação Mestrado). Disponível em http://www.repositorio.unb.br/handle/10482/10777

Ogińska-Bulik, N. (2014). Osobowość typu D a jakość życia osób po zawale serca. Kardiologia Polska, 72(7), 624-30. doi: 10.5603/KP.a2014.0066

Parker, J., \& Stahel, M. (2010). Password: K Dictionaries: English dictionary for Speakers of Portuguese. São Paulo, SP: Martins Fontes.

Pereira, M. da G., Pedras, S., \& Machado, J. C. (2013). Adaptação do questionário de confiança no médico em pacientes com diabetes tipo 2 e seus companheiros. Psicologia: Reflexão e Crítica, 26(2), 287-295. doi: 10.1590/S0102-79722013000200008

Petrie, K. J., \& Weinman, J. (2006). Why illness perceptions matter. Clinical Medicine, Journal of the Royal College of Physicians of London. Clinical Medicine, 6(6), 536-539. doi: 10.7861/clinmedicine.6-6-536

Poffo, M. R., Vieira De Assis, A., Fracasso, M., Miguel, O., Filho, L., Matos De Menezes Alves, S., ... Schmitt, C. B. (2017). Profile of Patients Hospitalized for Heart Failure in Tertiary Care Hospital. International Journal of Cardiovascular Sciences, 30(3), 189-198. doi: 10.5935/2359-4802.20170044

Riley, J. P., Habibi, H., Banya, W., Gatzoulis, M. A., Lau-Walker, M., \& Cowie, M. R. (2012). Education and support needs of the older adult with congenital heart disease. Journal of Advanced Nursing, 68(5), 1050-1060. doi: 10.1111/j.1365-2648.2011.05809.x

Salomão, L. Z., Almeida, R. M. de S. S. A., Mouzinho, I. T., Slaviero, R. S., Fernandes, Y. C. F., 
Dantas, G. C., \& Rodrigues, R. F. (2017). Análise dos pacientes submetidos ao cateterismo cardíaco e cineangiocoronariografia no período de 2012 a 2015 em hospital referência de ensino no município de Cascavel/PR. Revista Thêma et Scientia, 6(2E), 228-233. Disponível em http://www.themaetscientia.fag.edu.br/index.php/RTES/article/view/302

Seabra, C. R., Peuker, A. C. W. B., \& Castro, E. K. (2015). Modelo de Autorregulação em Saúde e câncer de mama: uma revisão sistemática de literatura. Saúde e Desenvolvimento Humano, 3(2), 79-105. doi: 10.18316/2317-8582.15.7

Sousa, S. M., Bernardino, E., Vicelli, R. M. M., \& Kalinowski, C. E. (2014). Perfil de pacientes submetidos ao cateterismo cardíaco: Subsídio para prevenção de fatores de risco cardiovascular. Cogitare Enfermagem, 19(2). doi: 10.5380/ce.v19i2.36984

Suguihura, A. L. M. (2014, June 3). Personalidade tipo D e doenças cardiovasculares: adaptação de uma escala (Dissertação de Mestrado, Universidade de São Paulo, Ribeirão Preto, SP, Brasil). doi: https://doi.org/10.11606/D.59.2014.tde-17072014-142034

Sumin, A. N., \& Raih, O. I. (2016). Influence of Type D Personality on Adherence to Treatment in Cardiac Patients. Kardiologiia, 56(7), 78-83. Disponível em http://www.ncbi.nlm.nih. gov/pubmed/28290911

Sumin, A. N., Raikh, O. I., Gaifulin, R. A., Korok, E. V, Bezdenezhnykh, A. V, Ivanov, S. V, \& Barbarash, O. L. (2015). Predisposition to Psychological Distress in Patients After Coronary Bypass Surgery: Relation to One Year Prognosis. Kardiologiia, 55(10), 76-82. Disponível em http://www.ncbi.nlm.nih.gov/pubmed/28294799

Sumin, A. N., Ră̌kh, O. I., Karpovich, A. V, Korok, E. V, Bezdenezhnykh, A. V, Bokhan, I. E., \& Barbarash, O. L. (2012). Personality types in patients with atherosclerosis of different localization: prevalence and clinical features. Klinicheskaia Meditsina, 90(4), 43-9. Disponível em http://www.ncbi.nlm.nih.gov/pubmed/22896980

Taddeo, P. da S., Gomes, K. W. L., Caprara, A., Gomes, A. M. de A., Oliveira, G. C. de, \& Moreira, T. M. M. (2012). Acesso, prática educativa e empoderamento de pacientes com doenças crônicas. Ciência \& Saúde Coletiva, 17(11), 2923-2930.doi: 10.1590/S1413-81232012001100009

Thomas, C. V., \& Castro, E. K. D. (2012). Personalidade, comportamentos de saúde e adesão ao tratamento a partir do modelo dos cinco grandes fatores: uma revisão de literatura. Psicologia, Saúde \& Doenças, 13(1), 100-109. Disponível em http://www.scielo. mec.pt/scielo.php?script=sci_arttext\&pid=S1645-00862012000100009\&Ing=pt\&tlng=es.

Tziallas, D., Kostapanos, M. S., Skapinakis, P., Milionis, H. J., Athanasiou, T., S Elisaf, M., \& Mavreas, V. (2011). The association between Type D personality and the metabolic syndrome: a cross-sectional study in a University-based outpatient lipid clinic. BMC Research Notes, 4(1), 105. doi: 10.1186/1756-0500-4-105

Urso-Junior, J. (2011, September 12). Stress e personalidade :" overview " e avaliação crítica de revisões sistemáticas sobre Padrão Comportamental Tipo A e Personalidade Tipo D com desfechos Versão Corrigida. (Tese de doutorado, Universidade de São Paulo, São Paulo, SP, Brasil).doi: 10.11606/T.47.2011.tde-30112011-103946

Van Dooren, F. E. P., Verhey, F. R. J., Pouwer, F., Schalkwijk, C. G., Sep, S. J. S., Stehouwer, C. D. A., ... Denollet, J. (2016). Association of Type D personality with increased vulnerability to depression: Is there a role for inflammation or endothelial dysfunction? - The Maastricht 
Study. Journal of Affective Disorders, 189, 118-125. doi: 10.1016/j.jad.2015.09.028

Wajner, A., Zuchinali, P., Olsen, V., Polanczyk, C. A., Rohde, L. E., Wajner, A.,. . Rohde, L. E. (2017). Causes and Predictors of In-Hospital Mortality in Patients Admitted with or for Heart Failure at a Tertiary Hospital in Brazil. Arquivos Brasileiros de Cardiologia, 109(4), 321-330. doi: 10.5935/abc.20170136

Wittkopf, P. G., Souza, C. A., Ferrari, E. P., Medeiros, T. E., \& Cardoso, F. L. (2016). Semelhanças entre comportamentos e papéis sociais apresentados por mulheres e homens com doença arterial coronariana. Arquivos de Ciências da Saúde, 23(2), 71-75. doi: 10.17696/2318-3691.23.2.2016.205

Yu, X., Chen, Z., Zhang, J., \& Liu, X. (2011). Coping mediates the association between Type $D$ personality and perceived health in Chinese patients with coronary heart disease. International Journal of Behavioral Medicine, 18(3), 277-84. doi: 10.1007/s12529-010-9120-y

Recebido em: 25/01/2018

Última revisão: 13/10/2018

Aceite em: 23/10/2018

\section{Sobre os autores:}

Natan Pinto do Nascimento - Psicólogo Residente em Cardiologia da Universidade de Passo Fundo. E-mail: natannascimento@live.com, Orcid: http://orcid.org/0000-0001-7472-4536

Gabriela Woll Dias - Psicóloga Residente no Programa de Residência Multiprofissional Saúde - Cardiologia pela Universidade de Passo Fundo. E-mail: gabrielawolldias@gmail.com, Orcid: http://orcid.org/0000-0001-5711-5974

Luciane Daroit - Graduação em Matemática e Mestrado em Ensino de Ciências Exatas pelo Centro Universitário Univates, Lajeado. Professora da Universidade de Passo Fundo. E-mail: ludaroit@upf.br, Orcid: http://orcid.org/0000-0002-5967-1605

Ciomara RibeiroSilva Benincá-Psicóloga Doutoraem Psicologia do Desenvolvimento pela Universidade Federal do Rio Grande do Sul. Professora na graduação e pós-graduação na Universidade de Passo Fundo. Tutora no Programa de Residência Multiprofissional em Cardiologia e Atenção ao Câncer da Universidade de Passo Fundo. E-mail: beninca@upf.br, Orcid: http://orcid.org/0000-0002-0242-2245 